Nota

\title{
SOFTWARE PARA DIMENSIONAMENTO DE IRRIGAÇÃO POR SULCOS'
}

\author{
Tarlei Arriel Botrel2; Patricia Angélica Alves Marques ${ }^{3,4 *}$ \\ ${ }^{2}$ Depto. Engenharia Rural - USP/ESALQ, C.P. 9 - CEP: 13418-900 - Piracicaba, SP. \\ ${ }^{3}$ Pós-Graduando do Depto. de Engenharia Rural - USP/ESALQ. \\ ${ }^{4}$ Bolsista FAPESP. \\ *Autor correspondente <pmarques@prudenet.com.br>
}

RESUMO: Considerando a importância da irrigação por sulcos e devido a este sistema de irrigação necessitar de trabalhosos cálculos e ensaios de campo para seu projeto, o presente trabalho teve como objetivo apresentar o software Sulcos 2000, para Windows 95, desenvolvido em linguagem Delphi 3.0. Este software auxilia a elaboração de projetos de irrigação por sulcos através de procedimentos de cálculos automáticos que agilizam e possibilitam testar várias situações durante a fase de projeto. Também permite a obtenção da equação de infiltração através do ensaio de campo, já a equação de avanço pelo ensaio de campo e pela simulação. À partir destas equações realiza-se os cálculos referentes ao projeto e fornece-se uma avaliação da irrigação. Os dados são salvos em um banco de dados que pode ser acessado posteriormente. O software apresenta relatórios impressos e acesso a tabelas e ferramentas de dimensionamento.

Palavras-chave: irrigação, sulcos, software

\section{SOFTWARE FOR THE CALCULATIONS OF FURROW IRRIGATION PROJECTS}

\begin{abstract}
Considering the importance of furrow irrigation, the need of difficult calculations and necessary field research for the project, this work developed a software 'Sulcos 2000', for Windows 95 in Delphi 3.0. This software assists and facilitates the elaboration of furrow irrigation projects through the procedures of automatic calculations that activate and allow to test several situations during the project phase. Infiltration equations are obtained from field research. Progress equations are obtained from field research and simulation. The data are saved in a database that can be accessed at any moment. The software also presents printed reports and allows access to tables and design tools.

Key words: irrigation, furrow, software
\end{abstract}

\section{INTRODUÇÃO}

A irrigação por sulcos é um método bastante antigo, largamente utilizado para culturas em linhas. É uma alternativa viável devido ao seu baixo custo de implantação e por apresentar menores custos operacionais, bem como baixa dependência da indústria de equipamentos de irrigação, facilitando assim seu uso pelo agricultor (Bernardo et al., 1977; Bernardo, 1995). Apesar de suas vantagens é um método pouco utilizado no Brasil, pois a elaboração de seu projeto é bastante trabalhosa e os profissionais da área, devido às dificuldades encontradas e à falta de incentivo de empresas, preferem optar por outros métodos de irrigação.

O projeto de um sistema de irrigação por sulcos é feito com base numa série de dados obtidos no local a ser irrigado, e um projeto mal conduzido, pode levar a resultados desastrosos, causando baixo rendimento da cultura e baixa eficiência de irrigação. Os níveis reduzidos de desempenho de irrigação por sulcos podem ser atribuídos ao dimensionamento incorreto e à operação e manejo insatisfatórios.
O método de irrigação por superfície, teve seu uso no Brasil iniciado no começo do século XX para cultivo de hortaliças, batatas, cana-de-açúcar e pomares nos Estados de Minas Gerais, Rio de Janeiro e São Paulo (Carvalho, 1984). O método de irrigação por sulcos, quando comparado a outros, ocupa área relativamente grande mundialmente. Hung (1986) relatou que dos 47.000 ha plantados com cana-de-açúcar pela Taiwan Sugar Corporation, 42.000 ha estavam sob irrigação por sulcos. Farmwide (1999) citaram que cerca de $90 \%$ do algodão da Austrália são irrigados e que quase a totalidade é irrigada por sulcos. Cackett (1984) comentou que a irrigação por sulcos era o método mais usado para irrigar cana de açúcar no Zimbabwe, onde aproximadamente $75 \%$ da cultura eram irrigados por sulcos e o restante, irrigado por aspersão. Segundo Santana et al. (1997) a irrigação por sulcos é o método que predomina nos perímetros irrigados de áreas públicas do Nordeste, originalmente administrados pelo DNOCS e CODEVASF. Segundo Orlando Filho (1983) a aplicação da vinhaça por sulcos é o sistema empregado por diversas usinas no Estado de São Paulo.

${ }^{1}$ Parte da Dissertação de Mestrado do segundo autor apresentada à USP/ESALQ - Piracicaba, SP. 
Os sistemas de irrigação superficial ou por gravidade, possuem como principal característica a distribuição de água na área a ser irrigada através da superfície do solo, utilizando a energia da gravidade. Para isto é exigida uma condição superficial adequada do solo, de modo a proporcionar um escoamento contínuo sem causar erosão.

Segundo Scaloppi (1986) e Frizzone (1993), os sistemas por superfície apresentam vantagens em relação aos outros sistemas de irrigação, podendo-se destacar as seguintes: geralmente apresentam o menor custo; maior simplicidade operacional, facilmente assimilada pelos irrigantes; adaptados a um grande número de solos e culturas; operação pouco afetada pelos ventos; elevado potencial para aumento de eficiência de irrigação e para redução do consumo de energia; não interfere nos tratamentos fitossanitários das culturas; permitem a utilização de águas com sólidos em suspensão ou poluídas; maior flexibilidade para superar eventuais interrupções operacionais e possibilidade de automação operacional. Scaloppi (1986) e Vieira (1995) relataram que à semelhança de outros sistemas de irrigação, também apresentam importantes limitações, tais como: dependência das condições topográficas; inadequados para solos muito permeáveis e pouco profundos; seu dimensionamento envolve ensaios de campo; reavaliações frequentes, com a finalidade de introduzir medidas dimensionais e operacionais corretivas; o sistema integra a área para a qual foi projetado, portanto, não pode ser deslocado para outras áreas; a cultura deve se adaptar ao sistema de irrigação; medidas efetivas de controle de erosão devem ser adotadas; limitada divulgação pela indústria e pelos técnicos.

Em contraste com outros métodos, a irrigação por sulcos não molha toda a superfície do solo, molha normalmente $30 \%$ a $80 \%$ da superfície total, reduzindo a formação de crostas na superfície dos solos argilosos e tornando possível realizar a colheita logo após as irrigações, o que não ocorre com outros métodos, exceto o de gotejamento (Kay, 1986; Bernardo, 1995).

Os parâmetros que afetam o desempenho da irrigação por sulcos são: a vazão da entrada, declividade do sulco, comprimento do sulco, coeficiente de rugosidade hidráulica, forma do sulco, lâmina de irrigação e características de infiltração do solo (Raghuwanshi \& Wallender, 1998; Andrade et al., 1998). As características de infiltração do solo, coeficiente de rugosidade e a forma do sulco são parâmetros que variam de local para local. Rezende et al. (1992) relataram que dentre os inúmeros fatores que afetam a irrigação por sulcos somente alguns apresentam um certo controle, sendo: vazão aplicada, comprimento das parcelas, espaçamento entre sulcos, tempo de aplicação da água e em alguns casos a declividade dos sulcos.

As eficiências de aplicação e de distribuição da água dos métodos superficiais são geralmente mais baixas que as dos métodos sob pressão, principalmente de solos de alta velocidade de infiltração. Para aumentar a eficiência da aplicação e uniformidade de distribuição por sulcos é recomendado a redução da vazão inicial (Rezende et al., 1988).

Segundo Baltra (1987) o cálculo computacional está abrindo um novo e vasto panorama com muitas vias de solução para os problemas da água e sua distribuição e benefício através da irrigação.

O presente trabalho teve como objetivo apresentar o software Sulcos 2000, para o ambiente Windows 95, desenvolvido em linguagem Delphi 3.0. Este auxilia e facilita a elaboração de projetos de irrigação por sulcos através de cálculos automáticos que agilizam e permitem testar várias situações durante a fase de projeto.

\section{MATERIAL E MÉTODOS}

Para a criação do Software Sulcos 2000 utilizouse da linguagem Delphi 3.0. Procurou-se uma interface agradável ao usuário, que permite abordar o mesmo projeto em várias situações e observar os resultados pelos parâmetros de eficiência. O programa foi construído com formulários específicos para cada módulo de ação, sendo: Formulário principal; Formulário de caracterização da propriedade; Formulário de obtenção das equações de avanço e de infiltração acumulada; Formulário de projeto; Formulário de redução de vazão; Formulário de avaliação da irrigação; Ferramentas; Formulário de relatório.

O formulário "Caracterização da propriedade" tem como objetivo obter a disponibilidade real de água do solo (DRA) e o turno de rega máximo (TR) a partir de dados do solo e da cultura. Com o valor da DRA, evapotranspiração de referência $\left(E_{0}\right)$ e o $K c$ da cultura calcula-se o turno de rega máximo (equação 1). A partir deste o usuário deverá escolher o turno de rega adotado e com este valor o programa calcula a lâmina de irrigação necessária (LL) pela equação 2.

$$
\begin{aligned}
& \mathrm{TR}=\frac{\mathrm{DRA}}{\mathrm{ET}_{\mathrm{O}} \cdot \mathrm{Kc}} \\
& \mathrm{LL}=\mathrm{TR} \cdot \mathrm{ET}_{\mathrm{O}} \cdot \mathrm{Kc}
\end{aligned}
$$

sendo:

TR - turno de rega (dias);

DRA - disponibilidade real de água do solo $(\mathrm{mm})$; $E T_{0}$ - evapotranspiração de referência $(\mathrm{mm} / \mathrm{dia})$;

Kc - coeficiente da cultura;

LL - lâmina líquida (mm).

No formulário que trata da obtenção das equações necessárias ao dimensionamento e avaliação do sulco, utilizou-se o método da análise de regressão demonstrado em Bernardo (1995) para os dados do ensaio de campo tratados por transformação logarítmica, obtendo assim os parâmetros da equação. No formulário de obtenção da 
equação de infiltração acumulada foram programadas duas metodologias de ajuste da equação de infiltração acumulada. A primeira é a obtenção da equação tipo potencial de Kostiakov pela regressão direta dos dados de campo e a segunda é a obtenção da equação de Kostiakov-Lewis com o parâmetro de velocidade de infiltração básica (fo). A equação de infiltração acumulada é obtida pela integração da equação de velocidade de infiltração (Criddle et al., 1946; Bernardo, 1995). A equação de velocidade de infiltração pode ser obtida por ensaios de campo através do método de entrada e saída como descrito em Bourant (1957). Para estimar a equação do tipo Kostiakov-Lewis utilizou-se a metodologia descrita por Frizzone (1993).

A equação de avanço pode ser obtida de duas maneiras: através de ensaio de campo ou por simulação. Nos dados de campo, é aplicada a análise de regressão, descrito em Olitta (1984). Para a simulação da fase de avanço utilizou-se o modelo SAVIS (Andrade et al., 1998) onde, com o tempo de avanço acumulado para cada segmento, ajustou-se por regressão os parâmetros da equação avanço.

No formulário de concepção do Projeto calculouse o tempo de oportunidade, o tempo de avanço e 0 comprimento máximo do sulco. O tempo de oportunidade (T) necessário para infiltração dessa lâmina é calculado pela equação 3.

$$
\mathrm{T}=\left(\frac{\mathrm{LL}}{\alpha}\right)^{\frac{1}{\beta}}
$$

sendo:

T - tempo de oportunidade (minutos); $\alpha ; \beta$ - parâmetros empíricos da equação de infiltração acumulada.

Quando se utilizou e equação de infiltração acumulada de Kostiakov-Lewis, tornou-se necessário utilizar do método numérico de Newton-Raphson para solucionar a equação e obter o tempo de oportunidade para infiltrar a lâmina necessária. A partir deste tempo de oportunidade calculou-se o tempo de avanço de acordo com Criddle et al. (1946) que indicam que o tempo de avanço deve ser igual ou menor que $1 / 4$ do tempo de oportunidade. Com este tempo de avanço e a equação de avanço obteve-se o comprimento máximo do sulco. O usuário deverá definir o comprimento adotado para o projeto. Com o comprimento adotado pelo usuário é recalculado o tempo de avanço e o tempo de total da irrigação, e então é realizada a seqüência de avaliação da irrigação.

Para o cálculo da vazão reduzida $(\mathrm{Qr})$ utilizou-se a equação 4 onde foram consideradas as recomendações de Walker \& Skogerboe (1987), em que a vazão deve ser reduzida a um valor que acompanhe a velocidade básica de infiltração.

$$
\mathrm{Qr}=1,1 \cdot\left(\frac{\mathrm{fo} \cdot \mathrm{L} \cdot \mathrm{E}}{3600}\right)
$$

sendo:

$$
\begin{aligned}
& \text { Qr - vazão reduzida }(\mathrm{L} / \mathrm{s}) \text {; } \\
& \text { fo - velocidade de infiltração básica }(\mathrm{mm} / \mathrm{h}) \text {; } \\
& \text { L - comprimento do sulco }(\mathrm{m}) \text {; } \\
& \text { E - espaçamento entre sulcos }(\mathrm{m}) \text {. }
\end{aligned}
$$

O formulário de avaliação da irrigação é composto de rotinas, simulando estaqueamento de 10 em 10 metros, que auxiliarão nos cálculos da uniformidade da distribuição de água e da eficiência da aplicação, podendo quantificar as perdas por drenagem no final do sulco, perdas por percolação e lâmina média aplicada. Segundo Bernardo et al. (1977); Reddy \& Clyma (1981) e Augusto et al. (1985) a lâmina média aplicada (ya) no sulco para vazão constante e para vazão reduzida, pode ser calculada respectivamente pelas equações 5 e 6 , apresentadas a seguir.

$$
\begin{aligned}
& \text { ya }=\frac{\mathrm{TC} \cdot \mathrm{qo} \cdot 60}{\mathrm{~L} \cdot \mathrm{E}} \\
& \mathrm{ya}=\frac{(\mathrm{Ta} \cdot \mathrm{qo}+\mathrm{T} \cdot \mathrm{Qr}) \cdot 60}{\mathrm{~L} \cdot \mathrm{E}}
\end{aligned}
$$

sendo:

ya - lâmina média aplicada em cada sulco $(\mathrm{mm})$; TC - tempo total de aplicação de água no sulco (minutos); qo - vazão aplicada no sulco (L/s);

$\mathrm{T}_{\mathrm{a}}$ - tempo avanço (minutos).

A lâmina média infiltrada no sulco (ym) foi calculada pela média das lâminas infiltradas em todo o comprimento do sulco considerando estacas a cada 10 metros e utilizando a equação de infiltração acumulada. A Uniformidade de distribuição (UD) é a relação entre a lâmina mínima de água infiltrada (Ymínima) e a lâmina média de água infiltrada (ym) em todo sulco, ela avalia a variabilidade da lâmina de água infiltrada, sendo determinada pela equação 7 .

$$
\mathrm{UD}=\frac{\text { Ymínima }}{\mathrm{ym}} \cdot 100
$$

sendo:

UD - uniformidade de distribuição (\%);

Ymínima - lâmina mínima de água infiltrada ( $\mathrm{mm})$; ym - lâmina média de água infiltrada ( $\mathrm{mm})$.

Segundo Folegatti (1985) e Augusto et al. (1985) a eficiência de aplicação (Ea) é a estimativa da porcentagem do total de água aplicado na irrigação que é 
considerada útil às culturas. Como demonstrada em Silva et al. (1984) e Walker \& Skogerboe (1987) a eficiência de aplicação pode ser calculada pela equação 8 .

$$
\mathrm{Ea}=\frac{\mathrm{LL}}{\mathrm{ya}} \cdot 100
$$

sendo:

$$
\text { Ea - eficiência de aplicação (\%). }
$$

A eficiência de armazenamento (Es) é definida como a porcentagem da lâmina necessária na profundidade efetiva do sistema radicular (LL) que ficou armazenada em disponibilidade à cultura (Folegatti, 1985). Para irrigações sem déficit a eficiência de armazenamento foi considerada $100 \%$. A perda por percolação (Pp) pode ser calculada pela equação 9 demonstrada em Bernardo et al. (1977) e Walker \& Skogerboe (1987). A perda por escoamento superficial $(\mathrm{Pe})$ pode ser calculada pela equação 10.

$$
\begin{aligned}
& \mathrm{Pp}=\frac{\mathrm{ym}-\mathrm{LL}}{\mathrm{ya}} \cdot 100 \\
& \mathrm{Pe}=\frac{\mathrm{ya}-\mathrm{ym}}{\mathrm{ya}} \cdot 100
\end{aligned}
$$

sendo:

$$
\begin{aligned}
& \mathrm{Pp} \text { - perdas por percolação (\%); } \\
& \mathrm{Pe} \text { - perdas por escoamento superficial (\%). }
\end{aligned}
$$

A opção Ferramentas constou da construção de um menu que permite 0 acesso a calculadora do sistema, tabelas auxiliares e opções para dimensionamento de motobomba, tubulações, canais e sifões. Estas ferramentas não estão vinculadas ao banco de dados, portanto não são variáveis armazenadas no arquivo de projeto.

A opção de tabelas auxiliares foi construída com tabelas que pudessem auxiliar o usuário no decorrer do projeto, ela consta de 8 tabelas sendo: coeficiente de cultura - Kc; profundidade efetiva do sistema radicular; propriedades usuais do solo; comprimento e vazões máximas de sulcos; valores de inclinação do talude, velocidade máxima e coeficiente de Manning para canais; coeficientes de Hazen-Williams; coeficientes de Flamant; fator $\mathrm{F}$ de disponibilidade da FAO (Azevedo Neto \& Alvarez, 1973; Doorenbos \& Pruitt, 1984; Olitta, 1984; Vieira, 1995).

A opção de dimensionamento da motobomba fornece ao usuário um dimensionamento rápido durante o processo de projeto. A potência hidráulica e a potência nominal do motor elétrico são calculadas pelas equações encontradas em Azevedo Neto \& Alvarez (1973). No dimensionamento de canais são calculados a área, o perímetro molhado, o raio hidráulico e a velocidade onde se utilizou as equações demonstradas em Azevedo Neto \& Alvarez (1973) e Bernardo (1995). Para o dimensionamento de tubulações foram considerados 0 dimensionamento hidráulico e o econômico. Para o dimensionamento hidráulico utilizou-se da expressão de Flamant para diâmetro menor que $50 \mathrm{~mm}$ e HazenWilliams para diâmetro igual ou maior que $50 \mathrm{~mm}$. Para o dimensionamento econômico utilizou-se o método das tentativas simplificadas encontrado em Bernardo (1995). O dimensionamento econômico procurou encontrar 0 diâmetro que fornece a menor soma de custos fixos e variáveis.

Segundo Vieira (1995) a utilização de sifões para a alimentação dos sulcos, é muito prática e eficiente, sendo ainda o que melhor se adapta ao critério de vazão reduzida. Pela equação 11 encontrada em Frizzone (1993) pode-se estimar valores da vazão para sifões. A partir desta equação pode-se isolar a variável diâmetro, e assim obter o diâmetro teórico para a vazão desejada.

$$
\mathrm{Q}=\frac{0,65 \cdot \pi \cdot 1000 \cdot \mathrm{D}^{2} \cdot \sqrt{2 \cdot 9,81 \cdot \Delta \mathrm{H}}}{4}
$$

sendo:

$$
\begin{aligned}
& \mathrm{Q} \text { - vazão (L/s); } \\
& \mathrm{D} \text { - diâmetro (metros); } \\
& \Delta \mathrm{H} \text { - altura de carga (metros). }
\end{aligned}
$$

\section{RESULTADOS E DISCUSSÃO}

No programa "Sulcos 2000", obtido da compilação de 12.989 linhas de programação desenvolvidas orientadas a objeto, os dados utilizados no Projeto são salvos em um banco de dados interno, do tipo Paradox, para serem acessados e alterados a qualquer momento pelo usuário. O software apresenta uma precisão dos cálculos realizados com 13 casas decimais. O programa consta de um formulário principal (Figura 1) onde tem-se as opções de criação de novo arquivo, abertura de um arquivo salvo anteriormente, apagar um arquivo existente (Figura 2), entrar na ajuda do sistema (Figura 3) e a opção sair para finalizar 0 aplicativo.

$\mathrm{Na}$ parte superior dos formulários apresenta-se um menu que permite o acesso a todas as funções dos botões existentes no formulário e também o acesso as ferramentas auxiliares. Para auxiliar o usuário existe a opção de "Help" que acessa a ajuda geral do programa.

Ao se escolher pela criação de um novo projeto tem-se a janela de caracterização (Figura 4), onde são preenchidos dados da propriedade, da cultura e do solo. Neste formulário é solicitado ao usuário o turno de rega adotado, e a partir deste calcula-se a lâmina de irrigação 
necessária. Após a caracterização da propriedade seguese para o formulário de obtenção das equações (Figura 4). Neste formulário apresentam-se dois botões sendo o primeiro 'Determinação da equação de infiltração acumulada' e o segundo 'Determinação da equação de avanço' que encaminha o usuário a janela correspondente. Após serem definidas as equações estas serão mostradas no painel a direita do formulário de obtenção das equações.

No formulário de obtenção da equação de avanço, existem 3 opções para obtenção da equação de infiltração acumulada: a entrada direta dos parâmetros da equação, a entrada direta dos parâmetros da equação de velocidade de infiltração (Figura 5) e obtenção da equação de velocidade de infiltração através de ensaio de campo pelo método de entrada e saída (Figura 6). A cada nova escolha do método utilizado para determinar a equação,

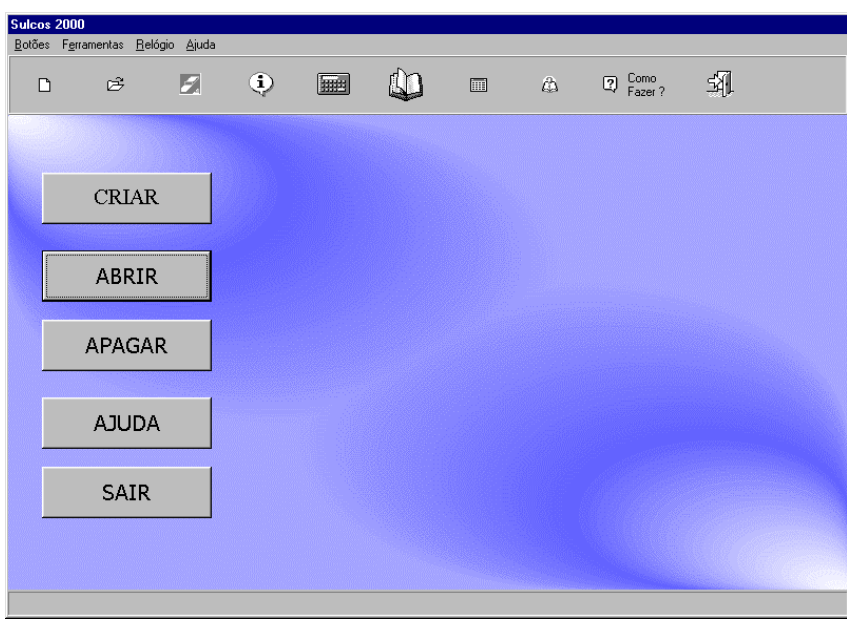

Figura 1 - Tela de entrada do programa Sulcos 2000. será exibido uma marca sobre a opção escolhida por último, demonstrando assim que esta será válida para o projeto e sua equação é mostrada na parte inferior do formulário.

De maneira similar, no formulário de obtenção da equação de avanço, existem 3 opções para obtenção da equação de avanço, sendo a entrada direta dos parâmetros da equação, obtenção através de ensaio de campo (Figura 7) e obtenção da equação de avanço pela simulação (Figura 8).

Com todas as equações já definidas, a tela de obtenção das equações estará completa seguindo desta para a tela de projeto (Figura 9), onde serão mostrados as equações vigentes no projeto, a

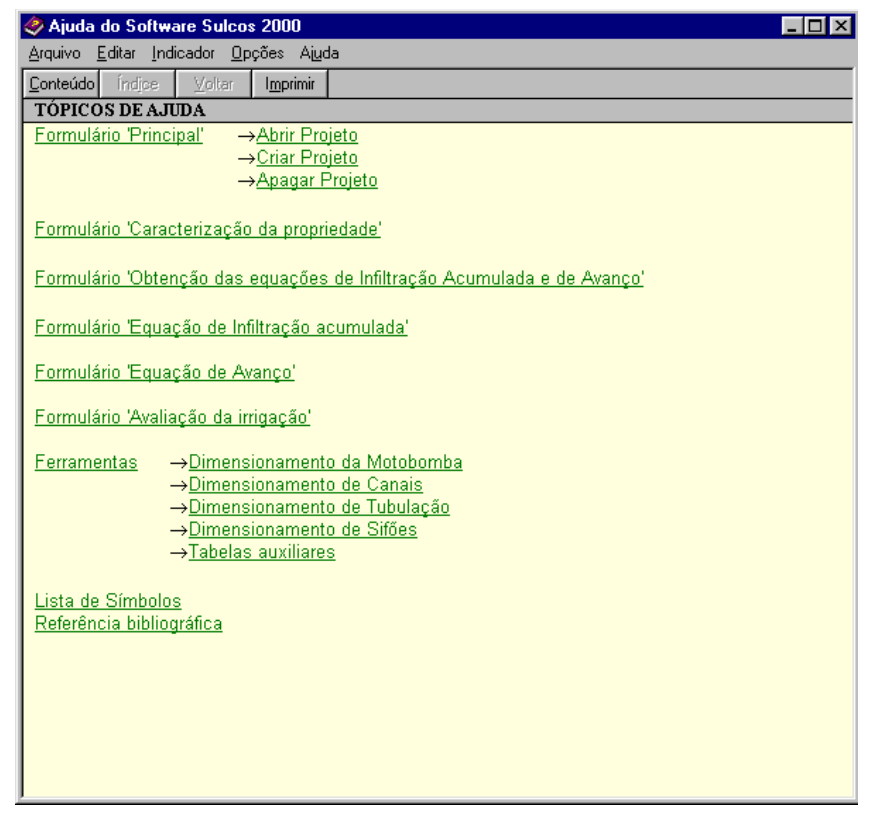

Figura 3 - Ajuda do Programa Sulcos 2000.


Figura 2 - Formulários para criar novo projeto, abrir projeto e apagar projeto. 
vazão de entrada, os tempos de infiltração e de avanço e o comprimento máximo para o sulco. O usuário deverá entrar com o comprimento adotado, a cada novo comprimento inserido será mostrado o resultado da avaliação.

No formulário de projeto tem-se a opção de visualização da avaliação (Figura 10), onde será mostrada uma planilha com as estacas simuladas de 10 em 10 metros, o tempo de avanço e lâmina infiltrada para cada estaca, bem como os parâmetros de avaliação e seus limites teóricos. Neste formulário há o botão
'Gráficos' que mostra os gráficos de infiltração acumulada, de avanço e da lâmina infiltrada ao longo do sulco (Figura 11).

No formulário de projeto tem-se a opção de redução de vazão que encaminha o usuário ao formulário de da vazão reduzida (Figura 12). A seguinte opção do formulário de projeto é o botão de relatório, que encaminha a um relatório com os dados de entrada e os parâmetros de eficiência do projeto, este relatório pode ser impresso ou salvo na forma TXT. No formulário de vazão reduzida (Figura 12) de maneira similar ao formulário projeto o usuário entra com a vazão
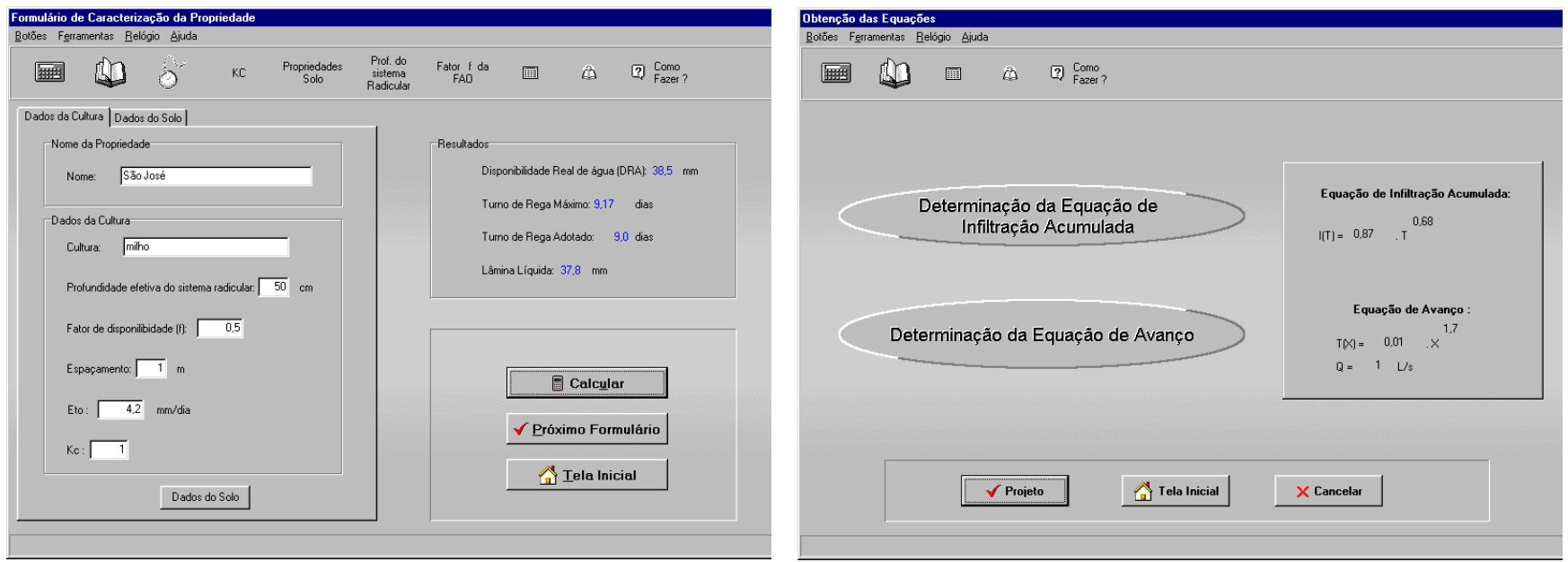

Figura 4 - Janela de caracterização e janela de obtenção das equações.

\section{Infiltração Acumulada}

Botões Ferramentas Bijuda

\begin{tabular}{|c|c|c|}
\hline 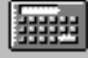 & 電 & $\theta$ \\
\hline
\end{tabular}

$$
\begin{aligned}
& \text { I(T) }=\boldsymbol{\beta} \cdot \mathrm{T}^{\boldsymbol{\alpha}}+\text { fo } \cdot \mathrm{T} \\
& \boldsymbol{\beta}=\longdiv { 0 , 8 7 } \\
& \boldsymbol{\alpha}=\longdiv { 0 , 6 8 } \\
& \text { fo }=\longdiv { 0 , 0 }
\end{aligned}
$$

I[T] = Infiltração acumulada (mm)

$\beta=$ coeficiente

$\alpha=$ coeficiente adimensional

$T=T$ empo (minutos)

fo = Velocidade de Infiltração básica $(\mathrm{mm} / \mathrm{h})$

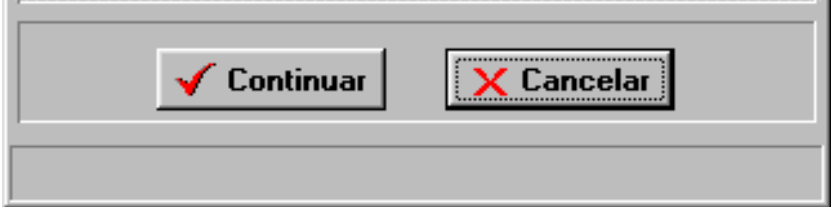

\section{Yelocidade de Infiltração}

Botōes Ferramentas Á Ajuda

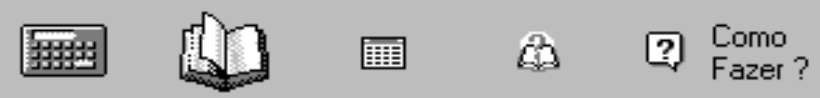

$$
\begin{aligned}
\mathbf{V}(t) & =\mathbf{a} \cdot \mathbf{T}^{\mathbf{n}}+\mathbf{f o} \\
\mathbf{a} & =\sqrt{35,78} \\
\mathbf{n} & =\sqrt{\frac{-0,32}{0,0}}
\end{aligned}
$$

VI(t $)=$ Velocidade de infiltração $(\mathrm{mm} / \mathrm{h})$

a e $n=$ coeficientes

$\mathrm{T}=\mathrm{Tempo}$ [minutos]

fo = Velocidade de Infiltração básica $[\mathrm{mm} / \mathrm{h}]$

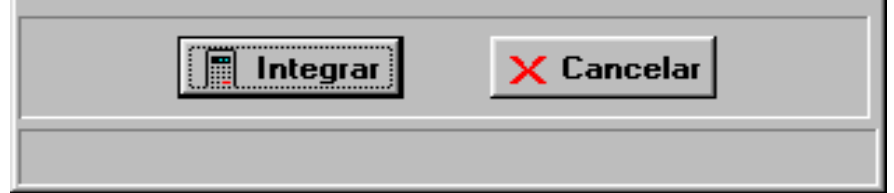

Figura 5 - Formulário de entrada direta dos parâmetros da equação de infiltração acumulada e dos parâmetros da equação de velocidade de infiltração. 
reduzida e obtêm os parâmetros de eficiência que são atualizados a cada nova vazão reduzida inserida. Apresenta também acesso ao formulário de avaliação e relatório.

O menu opção ferramentas apresentada nos formulários apresenta três opções, sendo: acesso a calculadora do sistema, tabelas auxiliares e dimensionamento. A ferramenta dimensionamento apresenta opção para dimensionar motobomba e tubulação (Figura 13), canais e sifão (Figura 14).

\section{CONCLUSÕES}

O Software Sulcos 2000 auxilia os técnicos a desenvolver projetos de irrigação por sulcos com facilidade, uma vez que as informações necessárias são solicitadas de modo interativo e possibilita criar várias situações durante a fase de projeto.

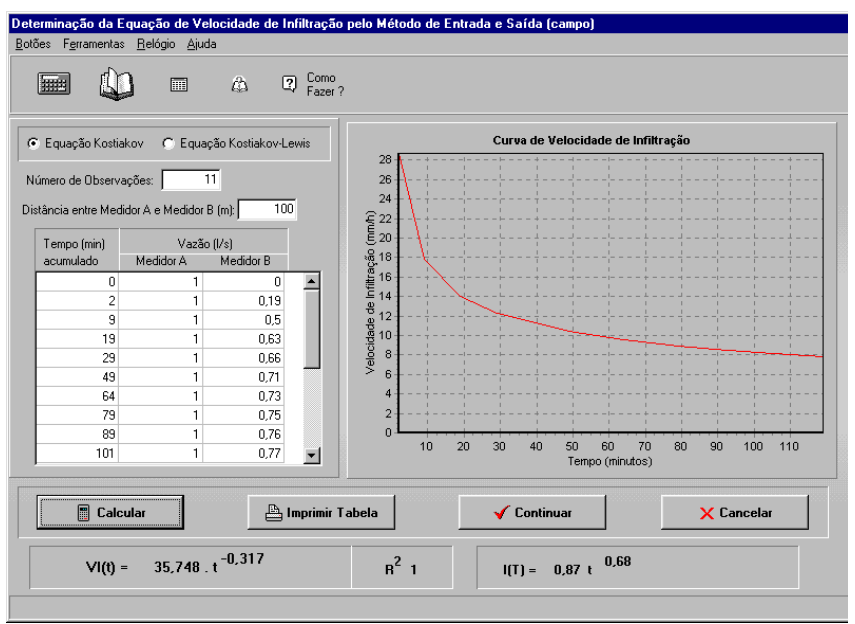

Figura 6 - Formulário de obtenção da equação de velocidade de infiltração pelo método de entrada e saída.

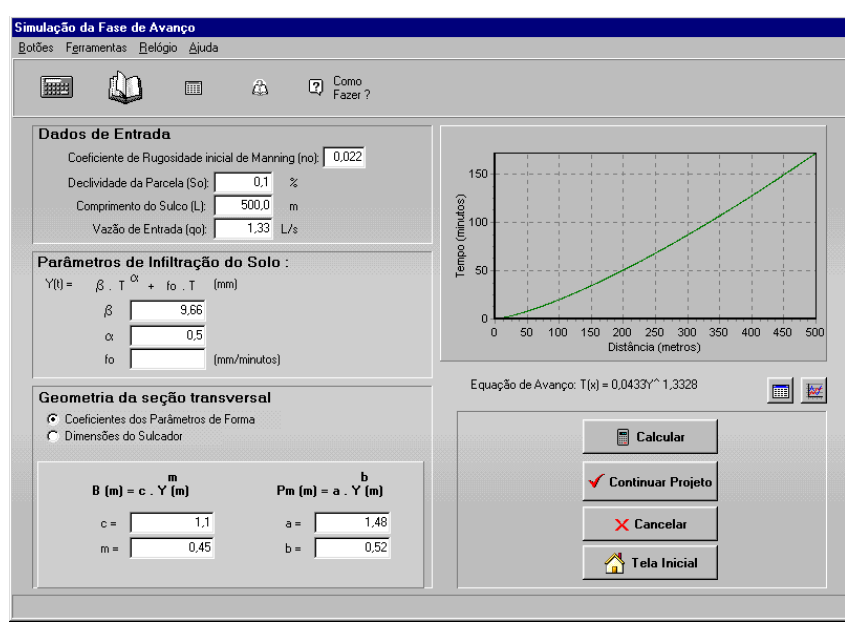

Figura 8 - Formulário de obtenção da equação de avanço por simulação.

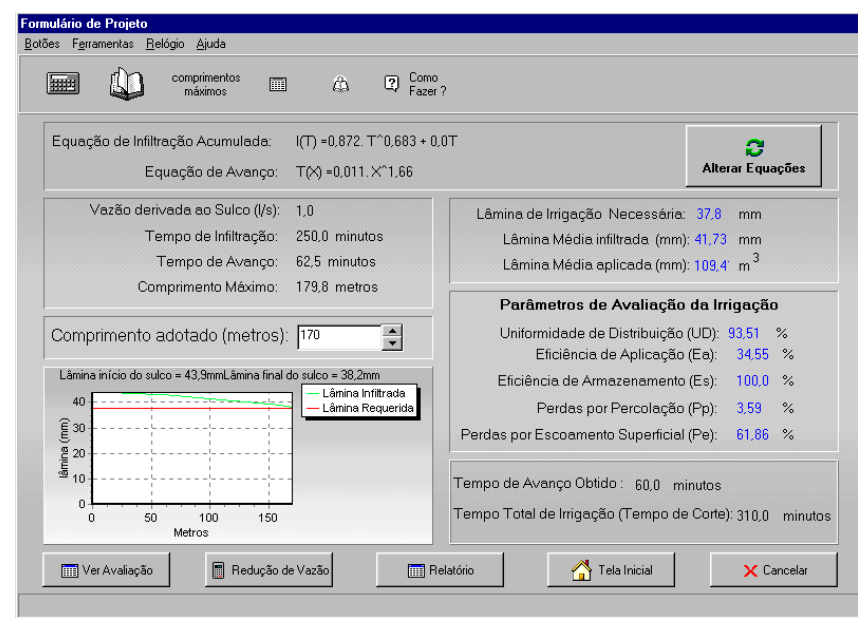

Figura 9 - Formulário de projeto.
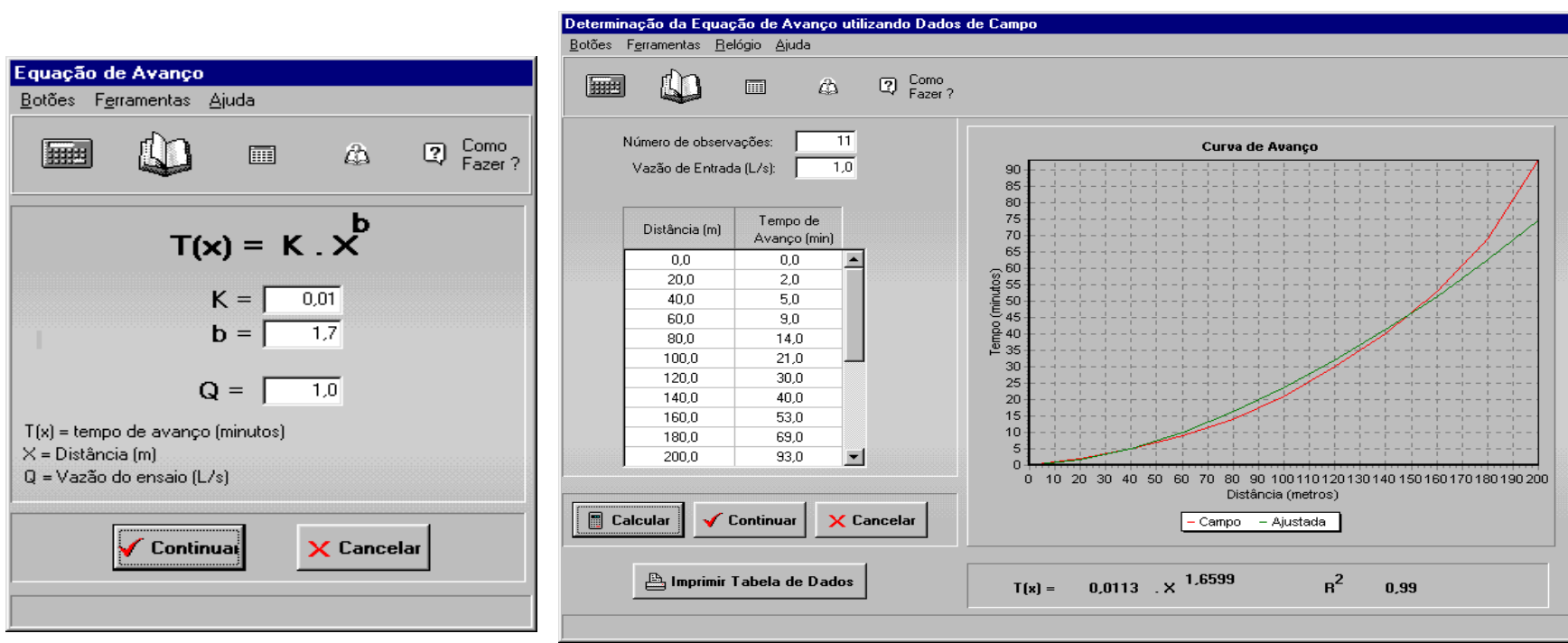

Figura 7 - Formulário de entrada direta dos parâmetros da equação de avanço e de obtenção da equação de avanço por ensaio de campo. 
O banco de dados mantém todos os dados do projeto armazenados em arquivo, o que permite ao projetista utilizá-los e economizar tempo durante o projeto.

As ferramentas aceleram e facilitam os cálculos, agilizando a tomada de decisões.

A possibilidade de imprimir os relatórios, tabelas de dados e gráficos fornece ao projetista uma nova possibilidade de discussão e comparação entre as situações testadas.

\section{AGRADECIMENTOS}

À FAPESP, pelo apoio dado à elaboração do Projeto, com a concessão de Bolsa de Mestrado à Engenheira Agrônoma Patricia Angélica Alves Marques e auxílio financeiro para a execução do projeto.

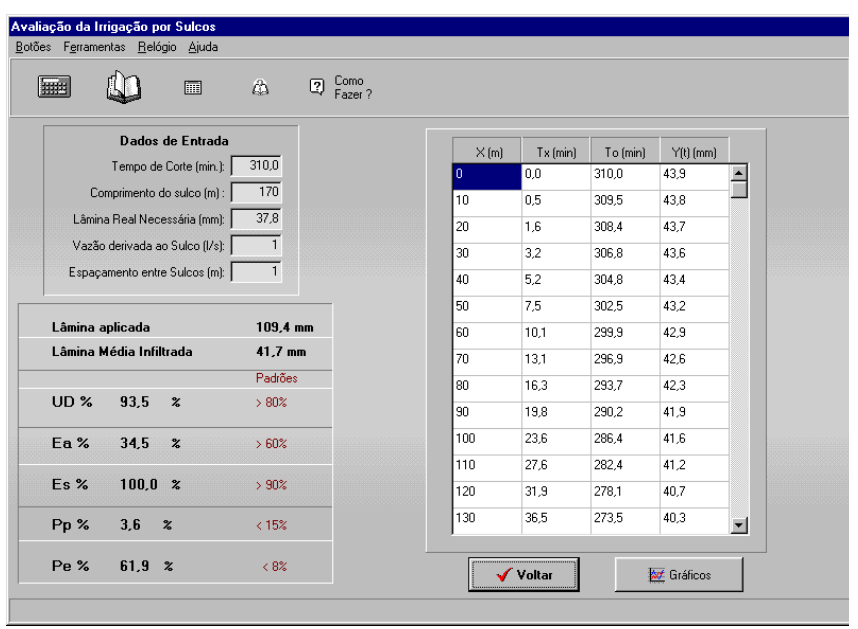

Figura 10 - Formulário de avaliação da irrigação.

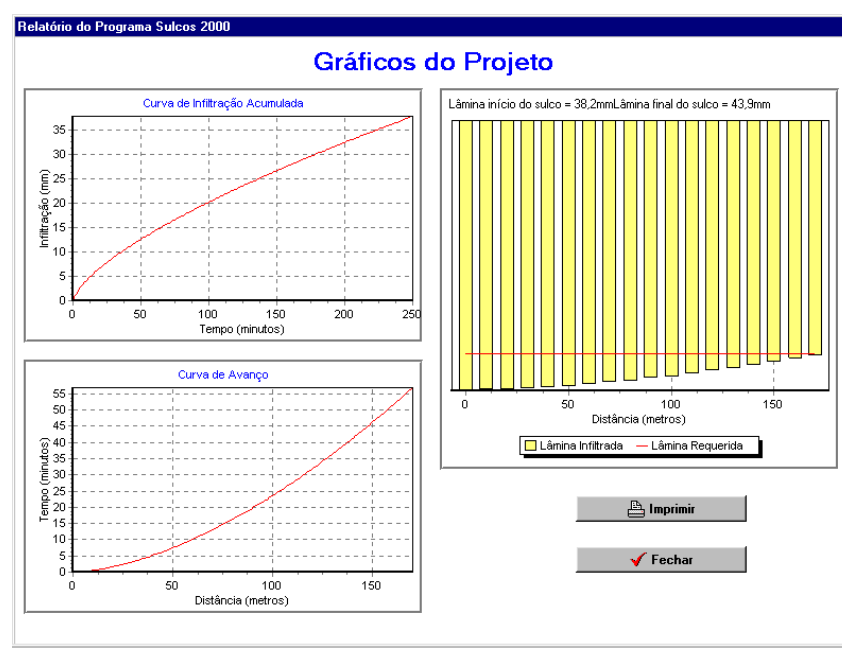

Figura 11 - Opção ‘Gráficos’ do formulário de avaliação da irrigação.

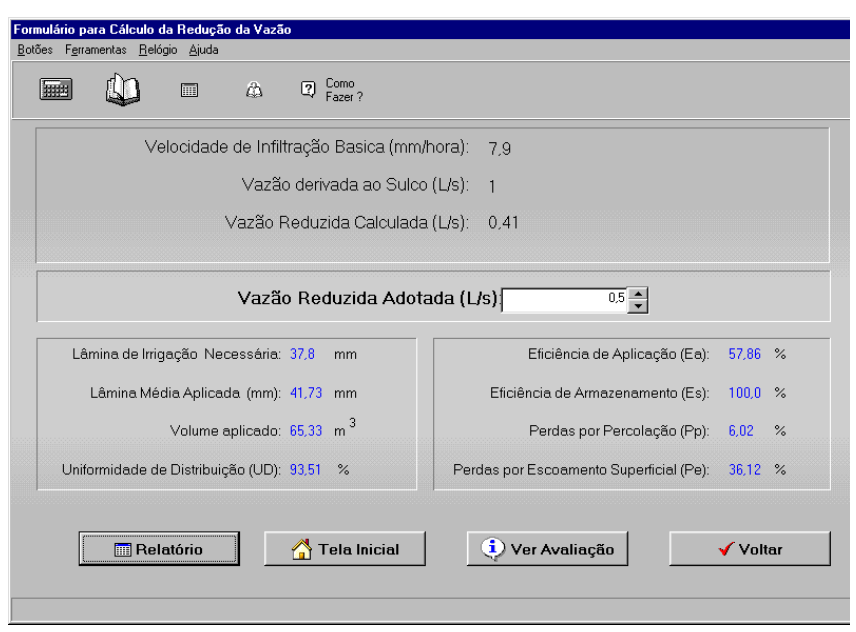

Figura 12 - Formulário da vazão reduzida.
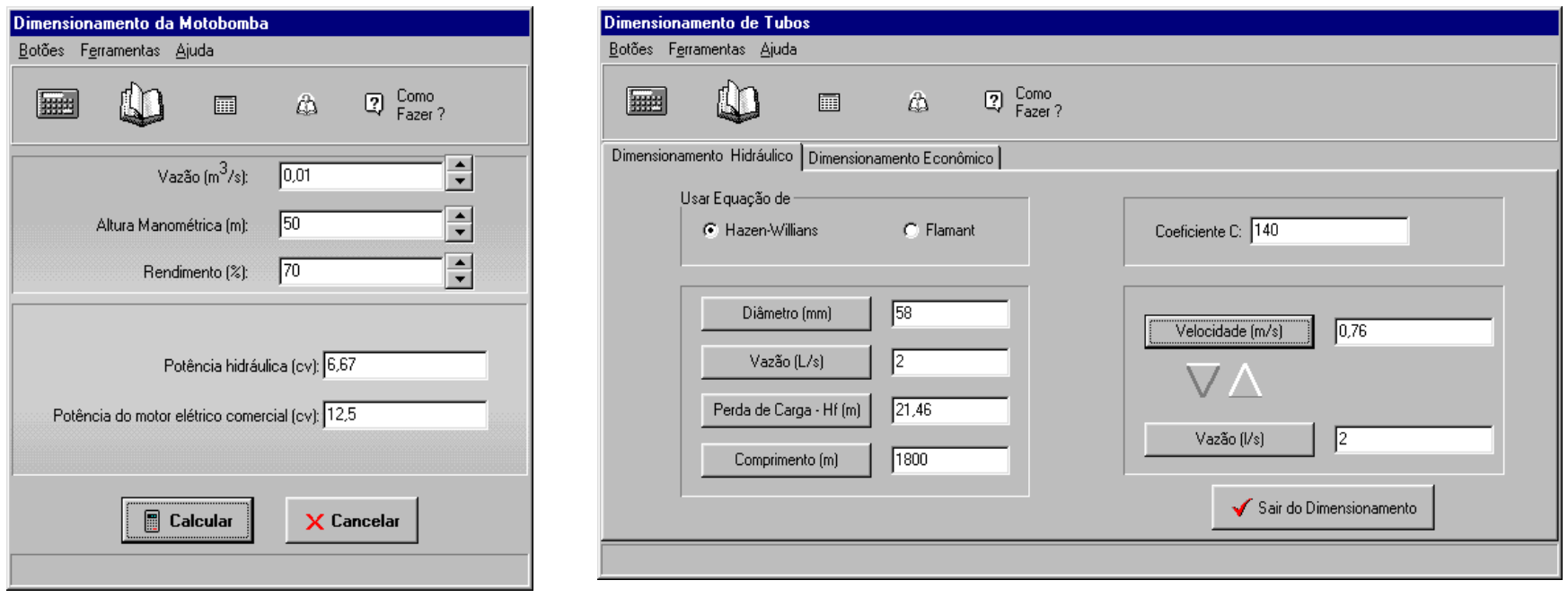

Figura 13 - Formulário de dimensionamento de motobomba e de dimensionamento de tubulação pelo método hidráulico. 

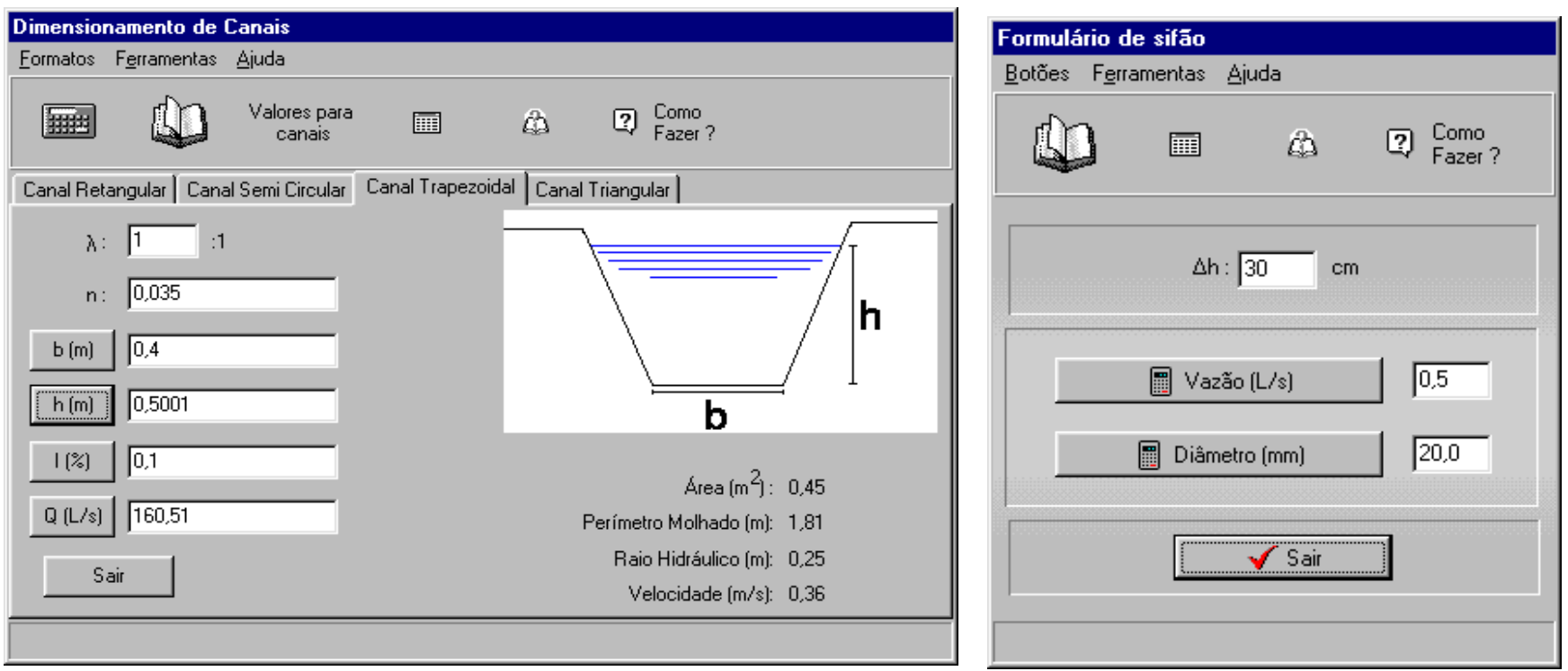

Figura 14 - Formulário de dimensionamento de canais e de dimensionamento de sifão.

\section{REFERÊNCIAS BIBLIOGRÁFICAS}

ANDRADE, D.V.; BOTREL, T.A.; PAZ, V.P.S.; FRIZZONE, J.A. Modelo matemático para simulação da fase de avanço na irrigação por sulcos. Revista Brasileira de Engenharia Agrícola e Ambiental, v.2, p.56-62, 1998.

AUGUSTO, S.G.; SOUZA, F.de; COSTA, J.O. Eficiência e uniformidade da água de irrigação por sulcos em declive. Engenharia Agrícola, v.9, p.7-18, 1985.

AZEVEDO NETO, J.M.de; ALVAREZ, G.A. Manual de hidráulica. 6.ed. São Paulo: Edgard Blücher, 1973. 2v.

BALTRA, A.O.C. Utilização da informática na irrigação. Irrigação e Tecnologia Moderna, n.28, p.13-15, 1987.

BERNARDO, S. Manual de irrigação. Viçosa: Imprensa Universitária, 1995. 657p.

BERNARDO, S.; RESENDE, M.; ARAÚJO, J.J. Eficiência de irrigação em sulco com redução da vazão inicial. Revista Ceres, v.24, p.261-277, 1977.

BOURANT, J.A. Developing furrow infiltrometer. Agricultural Engineering The Journal of ASAE, v.38, p.602-604, 1957.

CACKETT, K.E. Sugarcane irrigation handbook: a pratical guide to efficient irrigation management of sugarcane. Zimbabwe: ZSA Experiment Station, 1984. 90p.

CARVALHO, N.O. Irrigação no Brasil. Irrigação e Tecnologia Moderna, n.17, p.23-27, 1984.

CRIDDLE, W.; DAVIS, S.; PAIR, C.H.; SHOCKELY, D.G. Methods for evaluation irrigation systems. Washington: USDA, 1946. 24p. (Soil Conservation Service Agriculture Handbook, 82).

DOORENBOS, J.; PRUITT, W.O. Guidelines for predicting crop water requirements. Rome: FAO, 1984. (Irrigation and Drainage Paper, 24).

FARMWIDE Furrow irrigation. http://www.farmawide.com.au/ nff/cotton/education/furrow.html. (27 Dez. 1999).

FOLEGATTI, M.V. Desenvolvimento do Feijoeiro (Phaseolus vulgaris L.) submetido a diferentes lâminas médias de água através da irrigação por sulcos. Piracicaba, 1985. 99p. Dissertação (Mestrado) - Escola Superior de Agricultura "Luiz de Queiroz", Universidade de São Paulo.

FRIZZONE, J.A. Irrigação por superfície. Piracicaba: ESALQ, Depto. de Engenharia Rural, 1993. 183p. Sérè Didática, 5).

HUNG, H H. The sugar cane irigation improvem ent of Taiwan SugarCorporation Taiwan Sugar, v.33, p.19-23, 1986.
KAY, M. Surface irrigation: system and practice. Oxford: Cotswold, 1986. 143p.

OLITTA, A.F.L. Os métodos de irrigação. 11.ed. São Paulo: Nobel, 1984. 267p.

ORLANDO FILHO, J. Nutrição e adubação da cana-de-açúcar no Brasil. Piracicaba: IAA, 1983. 369p. (PLANALSUCAR, 2).

RAGHUWANSHI, N.S.; WALLENDER, W.W. Optimization furrow irrigation scheduling under heterogeneous conditions. Agricultural Systems, v.58, p.39-55, 1998.

REDDY, J.M.; CLYMA, W. Optimal design of furrow irrigation systems. Transactions of ASAE, v.24, p.617-623, 1981.

REZENDE, F.C.; SCALCO, M.S.; SCALOPPI, E.J.; SCARDUA, R. Alternativas de manejo e dimensionamento em irrigação por sulcos. In: CONGRESSO BRASILEIRO DE ENGENHARIA AGRÍCOLA CENEA/MA, 17., Iperó, 1988. Anais. Iperó: SBEA, 1988. p.102-114.

REZENDE, F.C.; FRIZZONE, J.A.; BOTREL, T.A. Otimização dos parâmetros de projeto de um sistema de irrigação por sulcos: II. análise da sensibilidade ao custo da água, da mão de obra e das estruturas hidráulicas. Engenharia Rural, v.3, p.32-49, 1992.

SANTANA, E.F.de; AZEVEDO, C.V.de; BARRETO, A.N.; NETO, J.D.; BEZERRA, J.R.C. Monitoramento sazonal do desempenho de um sistema de irrigação por sulcos no perímetro irrigado de São Gonçalo-PB. (compact disc) In: CONGRESSO BRASILEIRO DE ENGENHARIA AGRÍCOLA, 26., Campina Grande, 1997. Resumos. Campina Grande: SBEA, 1997.

SCALOPPI, E.J. Características dos principais sistemas de irrigação. Irrigação e Tecnologia Moderna, n.25, p.22-27, 1986.

SILVA, C.L.; VIANA, R.M.; SALES, J.G.C. Eficiência de irrigação por sulcos em um solo de cerrado. Irrigação e Tecnologia Moderna, n.17, p.27-33, 1984.

VIEIRA, D.B. As técnicas de irrigação 2.ed. São Paulo: Globo, 1995. 259p.

WALKER, W.R.; SKROGERBOE, G.V. Surface irrigation: theory and practice. New Jersey: Prentice-Hall, 1987. 386p.

$\overline{\text { Recebido em } 11.02 .00}$ 